\title{
DIRECTED HYPERGRAPH OBSERVERS-BASED QUALITATIVE FAULT MONITORING TASKS
}

\author{
Dhaou GARAI ${ }^{1}$, Rafika EL HARABI ${ }^{2}$, Faouzi BACHA ${ }^{1}$ \\ ${ }^{1}$ LISI Laboratory, INSAT / Carthage University, Tunisia, garaidhaou@ hotmail.com, faouzi.bacha@esstt.rnu.tn \\ ${ }^{2}$ Macs Laboratory, University of Gabes, Tunisia, rafikaharabi@yahoo.fr
}

Abstract

To create a precise model structure and perform fault monitoring algorithms for a wide range of complex systems along with dynamic behavioural characteristics, the causal graph-based methods were considered herein. In this paper, a new scheme was devised based on fast fault detection mechanism relying on the Directed Hypergraph Observer model.

The performance of the suggested pattern is illustrated by a case study including systems with single energy. Noting that the modern methods possess a large range of applications for the reliability and energy effectiveness analysis related to multi-energy systems. The Directed Hypergraph model architecture was exploited to generate the diagnostic condition based on a graphical observer.

Keywords: Graphical Modelling, Directed Hypergraph Observer, Fault monitoring, Qualitative analysis.

\section{INTRODUCTION}

Monitoring sophisticated industrial manufactories is an important task so as to insure the reliability, safety and maintenance of the required product quality [1]. This is notably real wherever regarding interconnections among productions, failures and maintenances. Complicated systems demand refined approaches to analyzing the failing impacts on the global system performance [2]. Recent reliability engineering always faces defiance related to the system description and model quantification.

In order to describe the dynamic behaviour of complex processes, the associated model can be an explicit mathematical set of equations (e.g state space representations) as already mentioned in [3, 4]. This type of representation generally involves a itemized information about the system characteristics and parameters to obtain a rigorous and precise model. In point of fact, the analytical interpretation has no correlation with the physical framework of the system. It considers the system as a black box without taking into account the energetic, structural and causal aspects of the state variables. Besides, it is tough to treat with the huge number of equations representing the dynamic behaviour of the various and interesting phenomena happening in the large scale systems together with the coupling of several energies known as complex systems. One manner to get away with these arduous problems is to imply the graphical approaches taken account of the energy transfer. Based on the structural properties and causal analysis of the energy transfer, graphical approaches (whether at a qualitative or quantitative level) are especially relevant for modelling engineering processes and improving Fault Detection and Isolation associated procedures (see $[5,6]$ and references therein for more details).

It is for this reason that the hypergraph formalism dedicated to reasoning in qualitative way is needed. The qualitative method rests on knowing rules and is further suitable when analytical models are hard to obtain or unavailable [7]. Hypergraphs are special well-respected as a generalization of graphs allowing an explicit description of the relationships. These tools are used in many areas of sciences $[8,9,10,11])$ and are even useful in engineering applications.

As being a unified graphical language founded on qualitative way, the hypergraph is approved to enhance the illustration of energy distributions over linked physical parts at any given time so as to obey the natural causality as underlined in $[12,13,14$, $15,16,17]$.

In the above-stated earlier works, a great deal of attention is paid to the system modelling problem. However, the graphs-based fault diagnosis scheme using the Directed Hypergraphs model was not introduced in the literature yet. Thus, an alternative framework for enhancing the FDI performances is required.

The motivation underlying this work was to sustain the structural notions and characteristics of a causal graphical modelling to improve fault detection targets. In this paper we focused on the hypergraph modelling used as a basis for the creation of a generic representation for the systems. Thereby, the graphical formalism should enable us 
to guarantee a systematic generation of information needed for system analysis and can then be used for fault diagnosis. To this end, a new form of residual generation was described based on the designed observer using the Directed Hypergraphs model.

The remainder of this paper is structured as follows. Section 2 introduced some necessary preliminaries and defined the problem statement. Section 3 was devoted to the main results related to the proposed fault monitoring framework using hypergraph observers to detect the actuator, sensor and physical faults. To prove the proposed model performance and efficiency, tutorial case studies were provide in section 4 . Section 5 presents a discussion and remarks of the graphical concept for the dynamic systems. Section 6 drew the main conclusions of the paper.

\section{PRELIMINARIES}

This section provided some elementary definitions involving hypergraphs and Directed Hypergraphs. The interested reader can refer to [7, $18,19]$ for further fundamental details associated to the theory of hypergraphs.

\subsection{Definition 1: (Hypergraph)}

Hypergraphs are an ordinary generalization of undirected graphs in which edges may be connected to any number of vertices (i.e. may contain more than two vertices). More exactly, a finite hypergraph $H=(V(H), \varepsilon(H))$ includes a finite set $V(H)$ and a family of subsets $\varepsilon(H)$ of non-empty subsets of $V(H)$. The component of $V(H)$ and $\varepsilon(H)$ are respectively called vertices and hyperedges, or simply edges of the hypergraph.

Throughout the present paper, we shall use the formal notation $H(V, \varepsilon)$ to denote the hypergraph $H . V=\left(v_{1}, v_{2} \ldots \ldots v_{n}\right)$ is the set of vertices and $\varepsilon$ $=\left(\varepsilon_{1}, \varepsilon_{2} \ldots \ldots \varepsilon_{m}\right)$ is the set of hyperedges where $\varepsilon_{i}$ $\subseteq v$ for $i=1 \ldots \ldots \ldots m$.

\subsection{Definition 2: (Sub-Hypergraph)}

A hypergraph $G$ filling $V(G) \subseteq V(H), \varepsilon(G) \subseteq$ $\varepsilon(H)$ is called a sub-hypergraph of $H$.

\subsection{Definition 3: (Directed Hypergraph)}

A directed hypergraph is a pair $(v, \varepsilon)$ where $v$ is a finite set of vertices and $\varepsilon \subseteq 2^{v} \times 2^{v}$ is a set of hyperarcs (hyperedges), such that each $\varepsilon=(T(\varepsilon)$ $, H(\varepsilon)) \in \varepsilon$ is an ordered pair of non-empty disjoint subsets $T(\varepsilon)$ and $H(\varepsilon)$; where $T(\varepsilon)$ and $H(\varepsilon)$ are the sets of vertices that appear respectively in the tail and the head of the hyperarc e. We say that e is an incident on each vertex in $T(\varepsilon) \cup H(\varepsilon)$, e is an outgoing hyperedge from $v$ and for each $v \in H(\varepsilon)$, e is an incoming hyperedge to $v$.

\section{DIRECTED HYPERGRAPH OBSERVERS BASED FAULT DETECTION SCHEMES}

The proposed fault detection architecture integrates the use of graphical observers to detect malfunctions at degraded components (sensors, actuators and system elements). This can be achieved by combining both of the cause and effect relationships and the qualitative reasoning. In this way, the observer model is systematically obtained from the Directed Hypergraph modelling and taken on thereafter for the qualitative analysis of the detection stage.

The suggested structure of the FD scheme is depicted in Fig. 1; where the principle is to estimate all the components of the state vector or, more generally, the system outputs, and, using the output estimation error of the observer, which are normally sensitive to faults, as the residuals of the FD system. This can be insured via a directed hypergraph observer. Therefore, the detection scheme must behave via assessing the residual indicator and then takes the decision. The main contributions of the paper may be summarised as follows:

- Synthesizing the generic hypergraph observers, the Directed Hypergraph Observer (DHO) based on the Directed Hypergraph model of the system (causal manipulations, structural observability....) therein.

- Presenting a new FD procedure based on a systematic approach by taking into consideration the theories of the qualitative reasoning and causal reasoning.

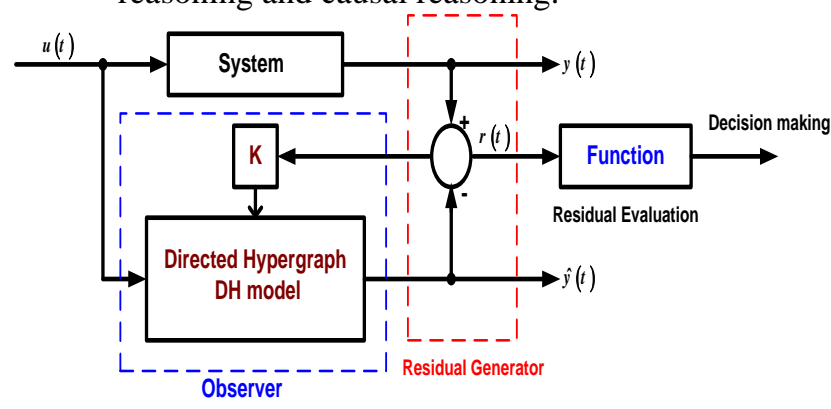

Fig. 1. Structure of the proposed FD system

\subsection{Directed Hypergraph Observers Design}

The Directed Hypergraph Observer (DHO) with proportional gain for linear systems is directly derived from the obtained hypergraph as following the below steps:

Step 1. Verify the presence of redundant outputs: the choice of non-redundant outputs can lead to compute the observer gain with the minimum dimensions. Nevertheless, it is even able to construct a Luenberger observer in spite of the existence of redundant outputs which does not affect observability. The computation becomes so easy and reduced when redundant outputs are eliminated. 
Step 2. Check the structural observability of the system: This later is represented through the Directed Hypergraph which is known as a universal depiction of the directed graph and identical to the bond graph description [21] [22]. From a hypergraphical viewpoint, Property 1 is used to verify the structural observability of the system in the linear or linearized part of the hypergraph pattern.

Property 1. (structural observability). The hypergraph model dedicate to depict a linear/linearized physical system is considered structurally observable if there is a causal hyperpath among the output of each causal hyperpath, and if all causal energy processors inputs are linearly independent [20].

Step 3. Linear injection of the output: two different linear injections are added to the unknown variables of the dynamic components constituting the Directed Hypergraph Observer. This generic representation totally includes the correction terms $K_{i}(y-\hat{y})$ through hyperedges reaching each unknown variables with a power conjugate corresponding to the energy storage elements of the Directed Hypergraph Observer DHO model, as indicated in Figures 2 and 3, respectively.

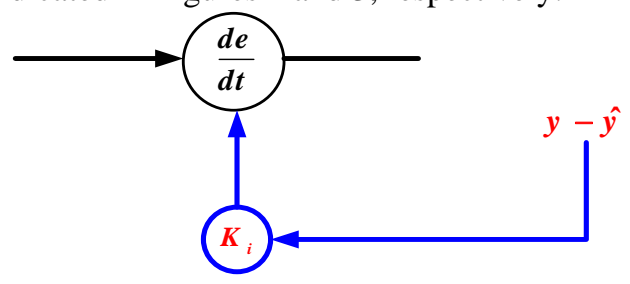

Fig. 2. C-element with a linear output injection in

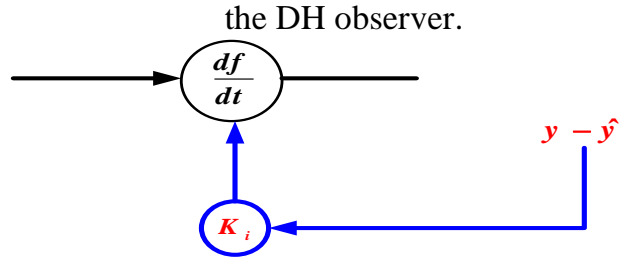

Fig. 3. I-element with a linear output injection in the DH observer.

Step 4. The Directed Hypergraph Observer gain calculation: To determine both transfer functions of the Directed Hypergraph model corresponding to the system and the associated DH observer, the causal manipulations are envisaged. In fact, the Directed Hypergraph Observer gain is directly calculated from the DH model via closed loops and causal hyperpaths regarding the Mason rule. Based on the classical pole placement methods, the characteristic polynomial of the desired polynomial $P_{d}(S)$ and the observer $P_{(A-K C)}(S)$ are identifying in order to get the gain.

Remark. A closed loop is defined as closed hyperpaths between the peaks of a hypergraph and a path of a hyperedge starting from the output of a node and returning to the input of the same node.

\section{CASE STUDY: HYDRAULIC SYSTEM}

Let us consider a simple Hydraulic System consisting of two tanks depicted in Figure 4, where $Q_{e}$ is the input flow, $Q$ and $Q_{s}$ are, respectively, the outputs flow in tank 1 and tank 2 and $\mathrm{h}_{1}$ and $\mathrm{h}_{2}$ are, respectively, the level-tank 1 and the level-tank 2 .

Figure 5 shows the Hamiltonian Bond Graph (HBG) model in integral causality of the hydraulic system .

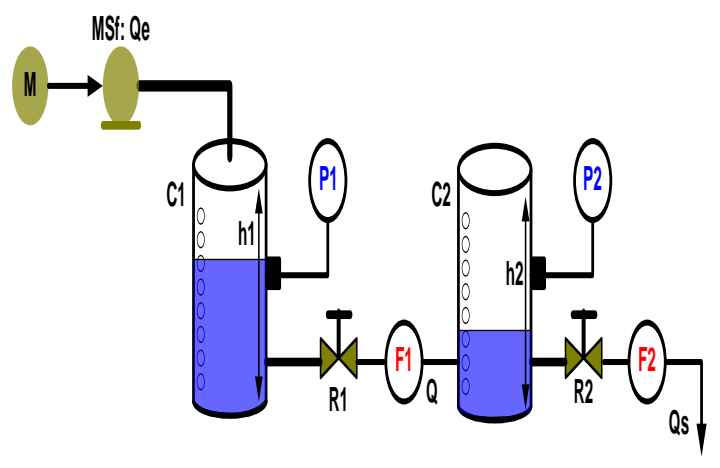

Fig. 4. Hydraulic system with two tanks

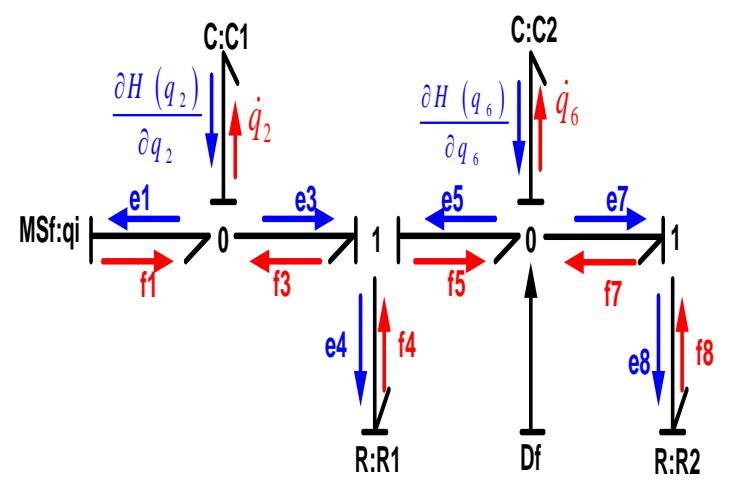

Fig. 5. Hamiltonian Bond Graph HBG Model in integral causality

This system is defined by the following differential equations:

$$
\left\{\begin{array}{l}
\left(c_{1}\right): C_{h 1} \frac{d P_{1}}{d t}=Q_{e}-Q \\
\left(c_{2}\right): C_{h 2} \frac{d P_{2}}{d t}=Q-Q_{s} \\
\left(c_{3}\right): Q=\frac{1}{R_{1}}\left(P_{1}-P_{2}\right) \\
\left(c_{4}\right): Q_{s}=\frac{1}{R_{2}} \cdot P_{2}
\end{array}\right.
$$

Such that $P_{1}=\gamma h_{1}$ and $P_{2}=\gamma h_{2}, C_{h 1}, C_{h 2}$ are respectively the hydraulic capacitance of tank 1 and tank 2 and $R_{1}, R_{2}$ are respectively, the hydraulic resistance of tank 1 and tank 2 .

The parameters for system are defined in table 1 . 
Table 1. Parameters for hydraulic system with two tanks

\begin{tabular}{ccc}
\hline Parameter & Symbol & Value \\
\hline Tank Section 1 & $C_{1}$ & $1 m^{2}$ \\
\hline Tank Section 2 & $C_{2}$ & $2 m^{2}$ \\
\hline $\begin{array}{c}\text { Hydraulic } \\
\text { Resistance }\end{array}$ & $R_{1}$ & $10 m\left(m^{2} s^{-1}\right)^{-1}$ \\
\hline $\begin{array}{c}\text { Hydraulic } \\
\text { Resistance }\end{array}$ & $R_{2}$ & $20 m\left(m^{2} s^{-1}\right)^{-1}$ \\
\hline
\end{tabular}

The Directed Hypergraph model $H_{d}=\left(V_{d}, \varepsilon_{d}\right)$ consists of seven vertices $V_{d}=\left\{Q_{e}, Q, Q_{s}, \dot{P}_{1}, \dot{P}_{2}, P_{1}\right.$ ,$\left.P_{2}\right\}$ and six hyperedges $\varepsilon_{d}=\left\{\varepsilon_{1}, \varepsilon_{2}, \varepsilon_{3}, \varepsilon_{4}, \varepsilon_{5}, \varepsilon_{6}\right\}$ where $\varepsilon_{1}=\left(\left\{Q_{e}, Q\right\},\left\{\dot{P}_{1}\right\}\right), \varepsilon_{2}=\left(\left\{\dot{P}_{1}\right\},\left\{P_{1}\right\}\right)$, $\varepsilon_{3}=\left(\left\{P_{1}, P_{2}\right\},\{Q\}\right), \quad \varepsilon_{4}=\left(\left\{Q, Q_{s}\right\},\left\{\dot{P}_{2}\right\}\right), \quad \varepsilon_{5}=$ $\left(\left\{\dot{P}_{2}\right\},\left\{P_{2}\right\}\right)$ and $\varepsilon_{6}=\left(\left\{P_{2}\right\},\left\{Q_{s}\right\}\right)$.

The hypergraph model $H=(V, \varepsilon)$ is made up of seven vertices $V=\left\{Q_{e}, Q, Q_{s}, \dot{P}_{1}, \dot{P}_{2}, P_{1}, P_{2}\right\}$ and six hyperedges $\varepsilon=\left\{\varepsilon_{1}, \varepsilon_{2}, \varepsilon_{3}, \varepsilon_{4}, \varepsilon_{5}, \varepsilon_{6}\right\}$ where $\varepsilon_{1}=\left\{Q_{e}, Q, \dot{P}_{1}\right\}, \varepsilon_{2}=\left\{\dot{P}_{1}, P_{1}\right\}, \varepsilon_{3}=\left\{P_{1}, P_{2}\right.$ $, Q\}, \varepsilon_{4}=\left\{Q, Q_{s}, \dot{P}_{2}\right\}, \varepsilon_{5}=\left\{\dot{P}_{2}, P_{2}\right\}$ and $\varepsilon_{6}=\left\{P_{2}\right.$, $\left.Q_{s}\right\}$.

Table 2. Hyperedge associated with the hypergraph model and Directed Hypergraph model

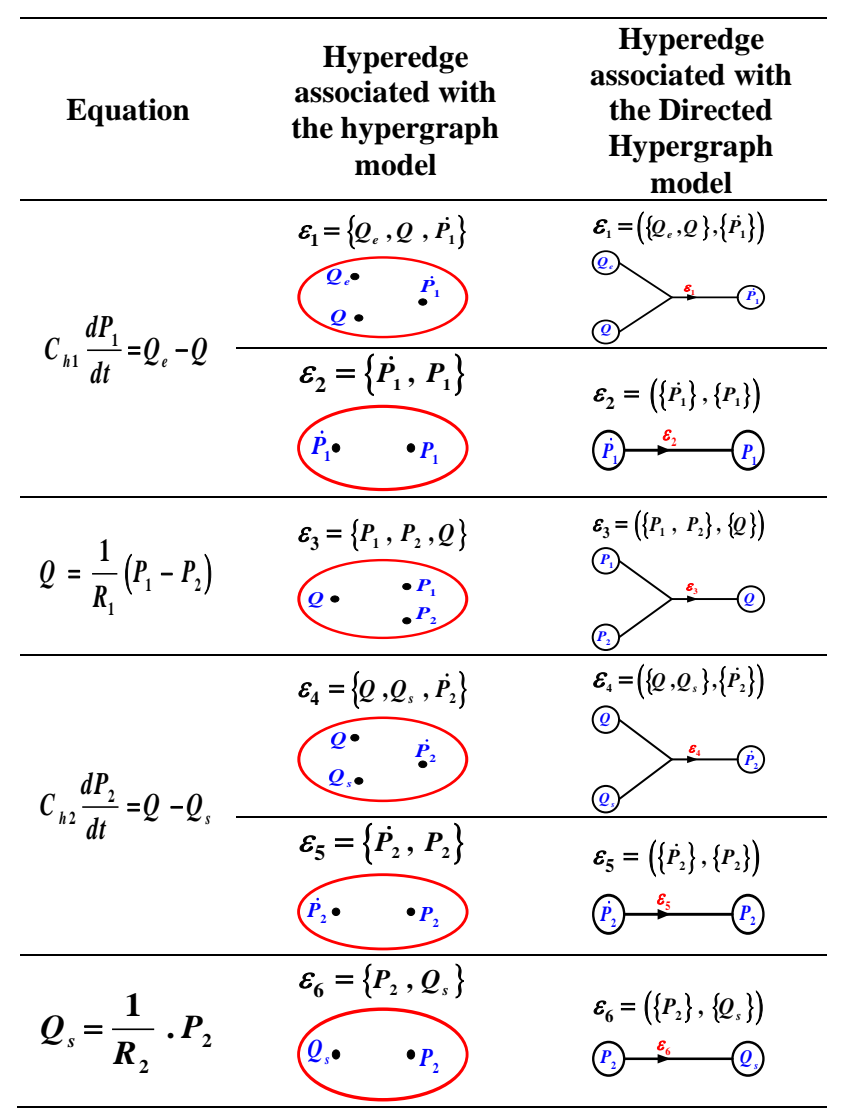

Based on Equation (1), the hypergraph models and Directed Hypergraph models of the hydraulic system are given in Figure (6) and Figure (7), respectively.

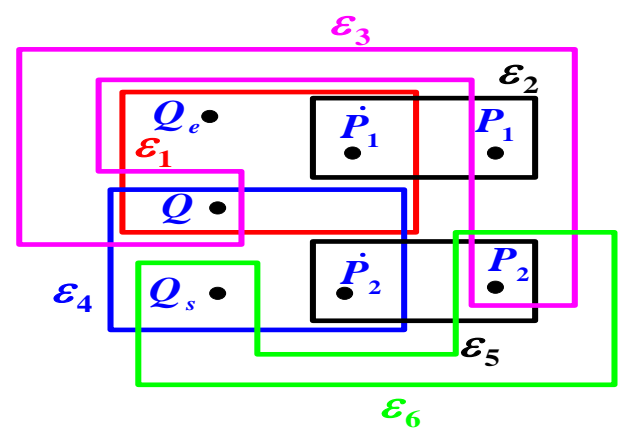

Fig. 6. Hypergraph model

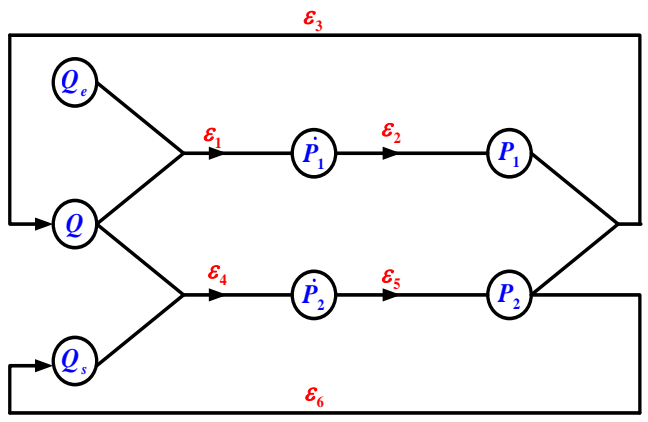

Fig. 7. Directed Hypergraph model

Before starting the design of the Directed Hypergraph Observer, as mentioned above the following steps must be validated:

Step 1. Verify the presence of redundant outputs:

The useless calculations can be kept away from by removing the redundant outputs, however, this step is omitted in this illustrative example since the number of detectors $D_{e}$ equal to the number of detectors $D_{f}$.

Step 2. Checking of the observability of the Directed Hypergraph model

This step depicts the stages to elaborate the Luenberger observer utilizing a Directed Hypergraph model. The algorithm is defined as follows:

- Unknown variables $U K=\left\{\dot{P}_{1}, \dot{P}_{2}\right\}$ in the system can be derived from the input and output variables:

$$
\left\{\begin{array}{l}
\dot{P}_{1}=\frac{1}{C_{1}}\left(Q_{e}-Q\right) \\
\dot{P}_{2}=\frac{1}{C_{2}}\left(Q-Q_{s}\right)
\end{array}\right.
$$

- There is a directed path from each unknown variable $U K=\left\{\dot{P}_{1}, \dot{P}_{2}\right\}$ arriving at the detectors. 


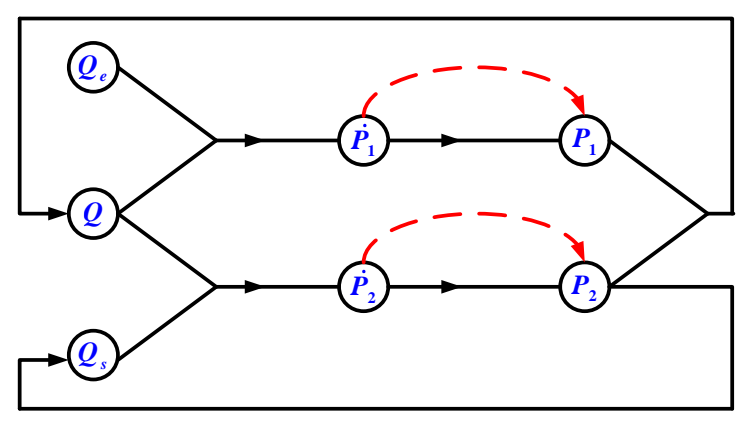

Step 3. Linear injection of the output:

The Directed Hypergraph Observer model can be depicted as shown in figure 8 .

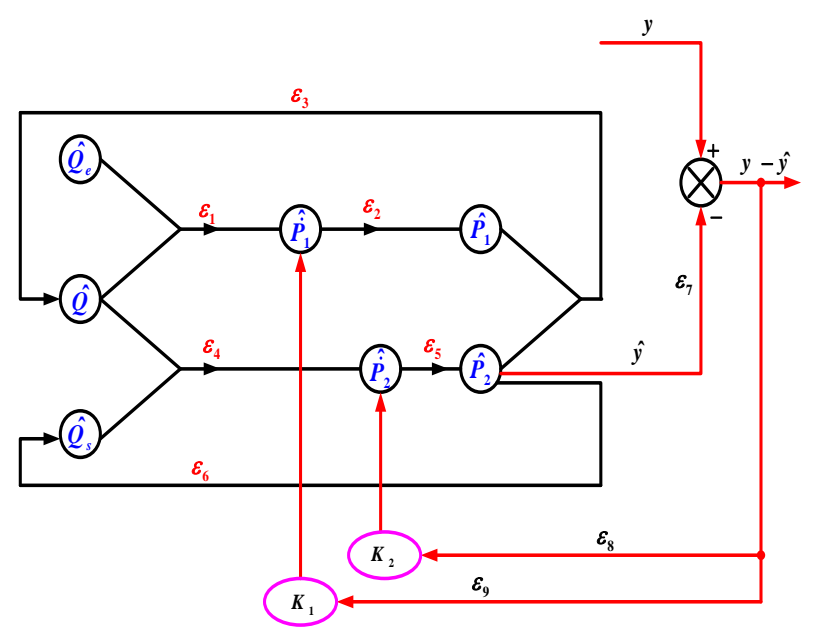

Fig. 8. Directed Hypergraph Observer model

Step 4: The Directed Hypergraph Observer gain calculation

The state variables of the system in figure 7 , are associated to two storage elements $\mathrm{C}$. The order of DH model is therefore, equal to two : $x=\left[\begin{array}{ll}x_{1} & x_{2}\end{array}\right]$

The characteristic polynomial of the state matrix is $P_{(A)}(s)$ calculated by the notions of closed loops and causal paths as shown in table 3 .

With $\varepsilon_{1}=\frac{1}{C_{1}}, \varepsilon_{3}=\frac{1}{R_{1}}, \varepsilon_{4}=\frac{1}{C_{2}}$ and $\varepsilon_{6}=\frac{1}{R_{2}}$

Loops 1 and 2 are disjoint. We then arrive at:

$$
\begin{gathered}
D(s)=1-\left(-\frac{1}{R_{1} C_{1} s}-\frac{1}{R_{2} C_{2} s}-\frac{1}{R_{1} C_{2} s}\right) \\
+\left(\frac{1}{R_{1} C_{1} s} \cdot \frac{1}{R_{2} C_{2} s}\right)
\end{gathered}
$$

The gain of the causal path is worth: $T_{1}(s)=$ $\frac{1}{R_{1} C_{1} C_{2} S^{2}}$ with $D_{1}=1$

So, we will have the transfer function :

$$
=\frac{H(s)}{s^{2}+\left(\frac{1}{R_{1} C_{1}}+\frac{1}{R_{2} C_{2}}+\frac{1}{R_{1} C_{2}}\right) s+\frac{1}{R_{1} R_{2} C_{1} C_{2}}}
$$

Thereby, the characteristic polynomial of the system is determined as :

$$
\begin{gathered}
P_{(A)}(s)=s^{2}+\left(\frac{1}{R_{1} C_{1}}+\frac{1}{R_{2} C_{2}}+\frac{1}{R_{1} C_{2}}\right) s \\
+\frac{1}{R_{1} R_{2} C_{1} C_{2}}
\end{gathered}
$$

Noting that the roots of the desired characteristic polynomial are selected to be rather faster than those of the model. $s_{d_{1}}=-0.31$ and $s_{d_{2}}=-0.032$ are respectively the values of the desired poles. Hence, the characteristic polynomial of the system becomes: $P_{d}(s)=s^{2}+0.35 s+$ 0.01

The characteristic polynomial $P_{(A-K C)}(S)$ in closed loop is calculated from the Directed Hypergraph Observer model (see figure 8) using the same procedure cited above as shown in table 4 $P_{(A-K C)}(s)=S^{2}+\left(\frac{1}{R_{1} C_{1}}+\frac{1}{R_{2} C_{2}}+\frac{1}{R_{1} C_{2}}+\frac{K_{2}}{C_{2}}+\right.$

$\left.\frac{K_{1}}{R_{1} C_{2}}\right) s+\left(\frac{1}{R_{1} R_{2} C_{1} C_{2}}+\frac{K_{2}}{R_{1} C_{1} C_{2}}\right)$ The identification between $P_{d}(s)$ and $P_{(A-K C)}(s)$ provides the gain values $K_{1}=-0.2$ and $K_{2}=0.35$

\section{Simulation results}

The initial condition of the DH model states are take into account null before carrying out the simulation of the different faulty scenarios on the Matlab toolbox MTIDS(Matlab Toolbox for Interconnected Dynamical Systems ). The graphical Luenberger observer of the DH model is given in the below diagram ( see figure 9 ).

Table 3. Closed loops gain for the DH model
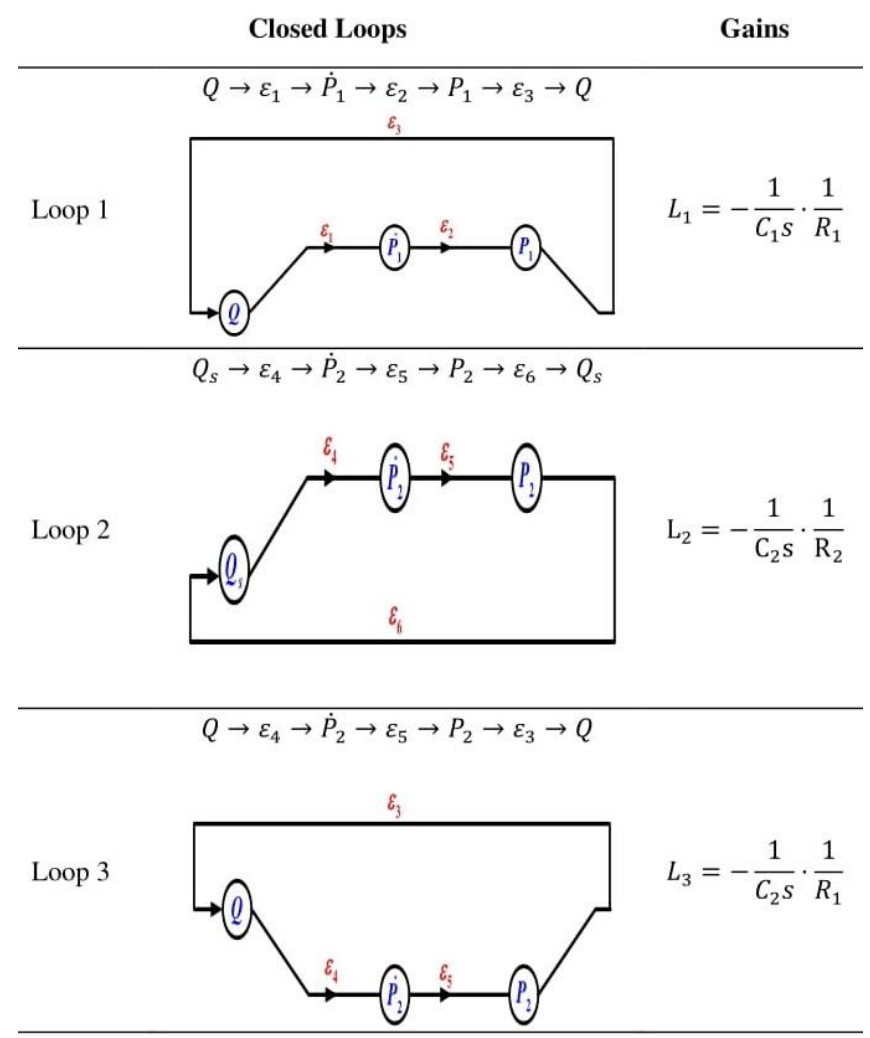
Table 4. Closed loops gain for the DHO model

\begin{tabular}{ccc}
\hline & Closed Loops & Gains \\
\hline Loop 1 & $\hat{Q} \rightarrow \varepsilon_{1} \rightarrow \hat{P}_{1} \rightarrow \varepsilon_{2} \rightarrow \hat{P}_{1} \rightarrow \varepsilon_{3} \rightarrow \hat{Q}$ & $L_{1}=-\frac{1}{C_{1} s} \cdot \frac{1}{R_{1}}$ \\
\hline Loop 2 & $\hat{Q}_{s} \rightarrow \varepsilon_{4} \rightarrow \hat{\dot{P}}_{2} \rightarrow \varepsilon_{5} \rightarrow \hat{P}_{2} \rightarrow \varepsilon_{6} \rightarrow \hat{Q}_{s}$ & $L_{2}=-\frac{1}{C_{2} s} \cdot \frac{1}{R_{2}}$ \\
\hline Loop 3 & $\hat{Q} \rightarrow \varepsilon_{4} \rightarrow \hat{P}_{2} \rightarrow \varepsilon_{5} \rightarrow \hat{P}_{2} \rightarrow \varepsilon_{3} \rightarrow \hat{Q}$ & $L_{3}=-\frac{1}{C_{2} s} \cdot \frac{1}{R_{1}}$ \\
\hline & $\varepsilon_{4} \rightarrow \hat{P}_{2} \rightarrow \varepsilon_{5} \rightarrow \hat{P}_{2} \rightarrow \varepsilon_{7} \rightarrow \varepsilon_{8} \rightarrow K_{2}$ & \\
& $\rightarrow \hat{P}_{2}$
\end{tabular}
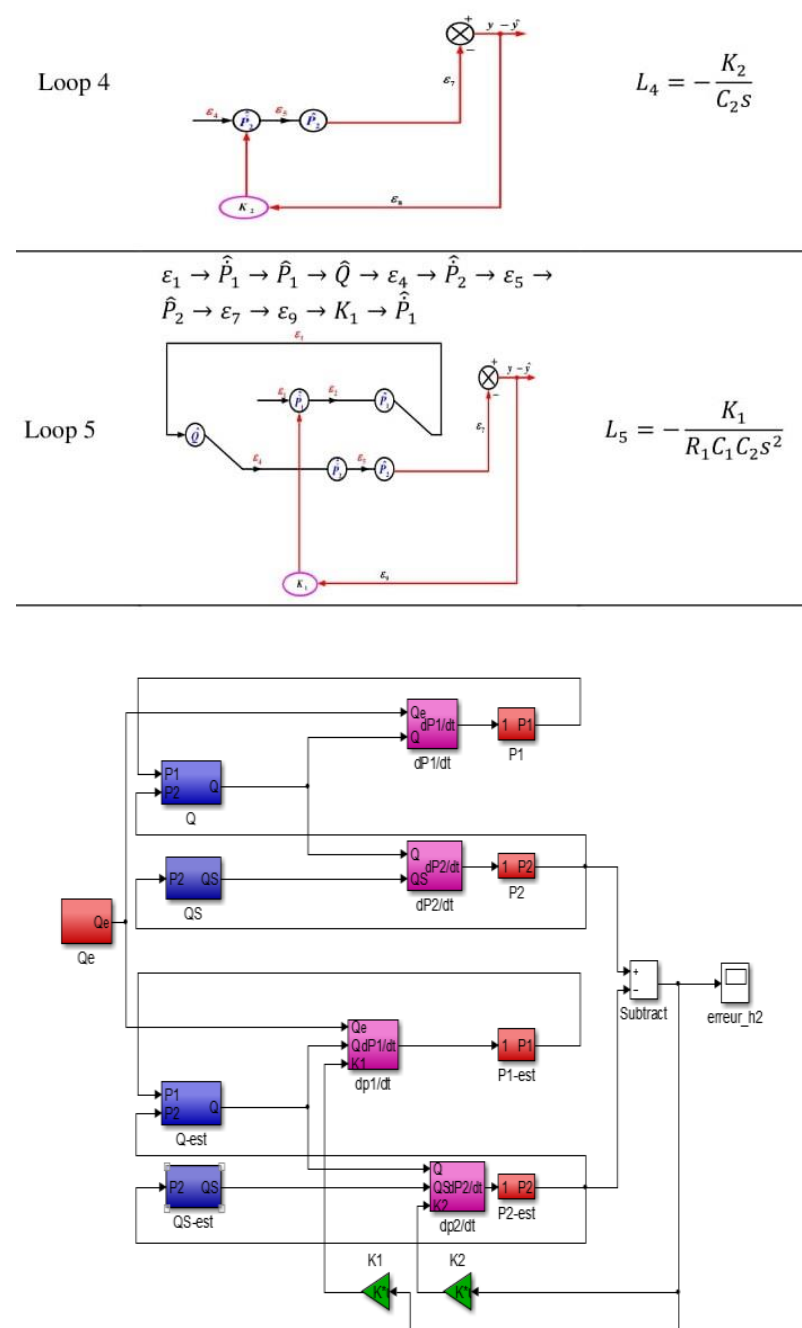

Fig. 9. Hydraulic system and its observer (DHO)

The numerical values of system Parameters Hydraulic system with two tanks are summarized in Table 1. The control input (input pump flow) is $Q_{e}$ $=0.1 \mathrm{~m}^{3} / \mathrm{s}$.

Figure 10 shows a clear precision in the estimation of the state variables of the system. We can see the paths of the state variable h1 and the state variable h2 (see figure 11) and the estimation error is null (see figure 12).

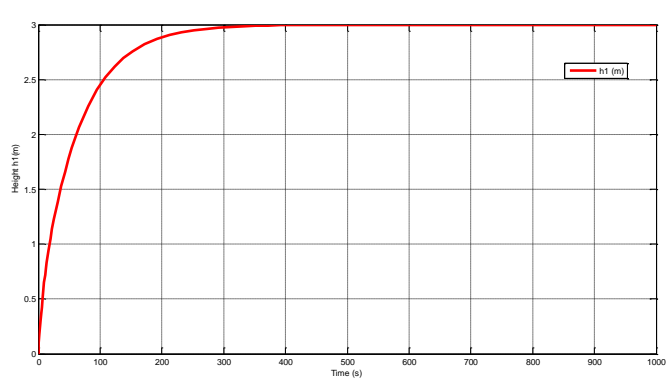

Fig. 10. State variable evolution h1

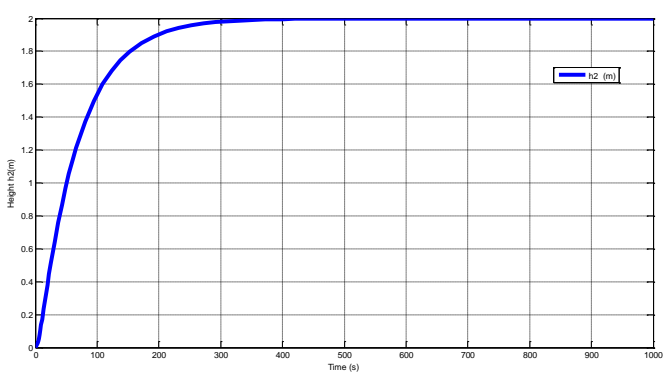

Fig. 11. State variable evolution h2

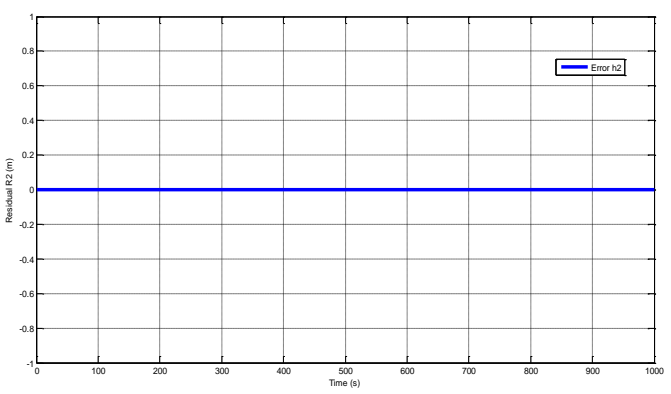

Fig. 12. Estimation error

So as to ensure the efficiency of the scheme dedicated to the fault detection issue using the graphical Luenberger observer, two fault scenarios were considered:

Actuator Failure: A pump failure.

Structural Failure: A water leak in tank 1.

- Scenario 1: Actuator failure

We considered now an actuator fault occurring between 400s and 500s. This fault affects the outputs, so, those lasts are drawn aside from their nominal values, see figures 13 and 14 .

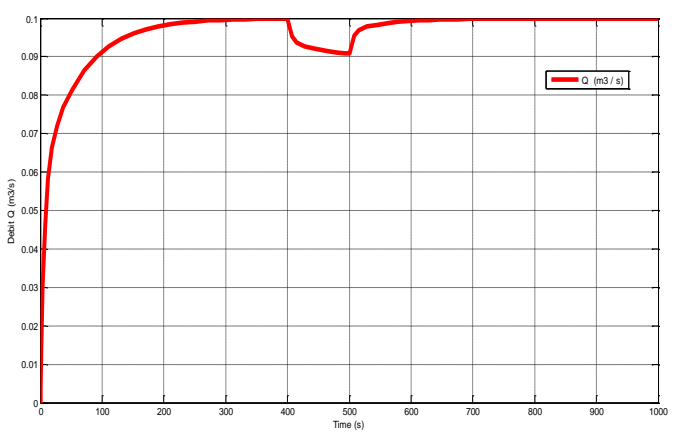

Fig. 13. Evolution of the output Q 


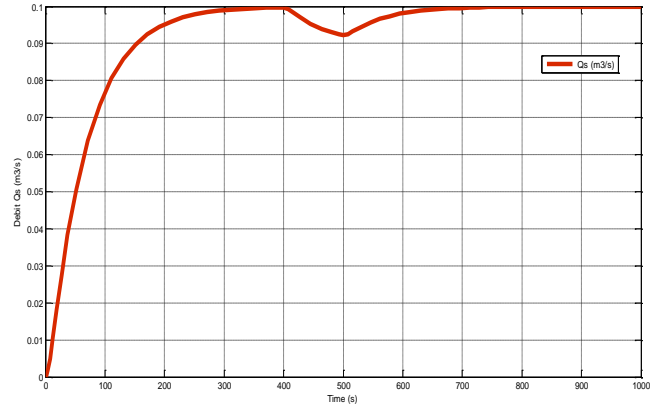

Fig. 14. Evolution of the output Qs

It is obviously easy to observe that, in faultyfree case, the residual signals are equal to zero, but, during the time interval $[400 s, 500 s]$, they were different from zero.

Besides, the actuator fault was detected at the same time of its triggering (see figures 15 and 16).

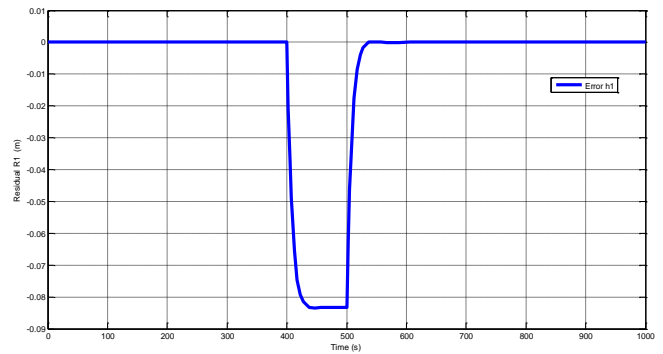

Fig. 15. Residual response R1

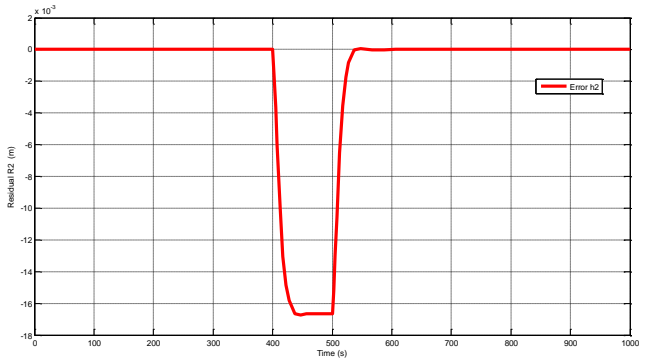

Fig. 16. Residual response R2

\section{- Scenario 2: Structural Fault}

The structural fault is represented by physically by the appearance of a leakage in the first tank. Indeed, the reduction of the water's height generates a fall of the pressure as well as the flow. The appearance of the structural failure of amplitude equal to $-0.01 \mathrm{~m}^{3} \mathrm{~s}^{-1}$ at instant $400 \mathrm{~s}$ (see Figures 17 and 18).

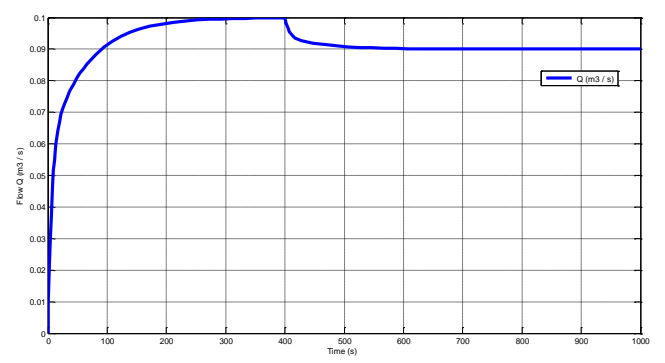

Fig. 17. Response of Q in the case of a structural failure

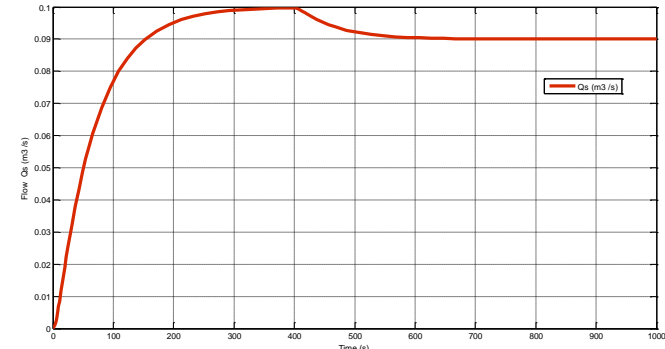

Fig. 18. Response of Qs in the case of a structural failure

Figure 19 shows a clear precision of estimation of the state variables of the system. We can clearly see the paths of the state variable $\mathrm{h} 1$ and the state variable h2 (see figure 20).

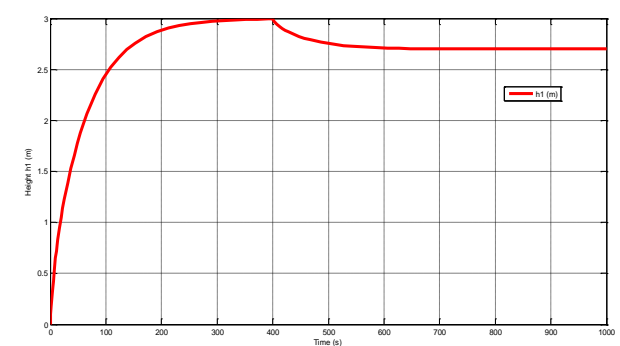

Fig. 19. State variable evolution $\mathrm{h} 1$ in the case of a structural failure

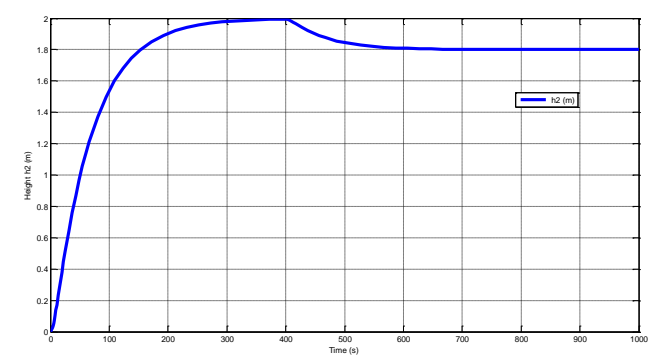

Fig. 20. State variable evolution h2 in the case of a structural failure

The dynamics of the system has changed and the residues are different from zero from the moment 400s as shown in figures 21 and 22.

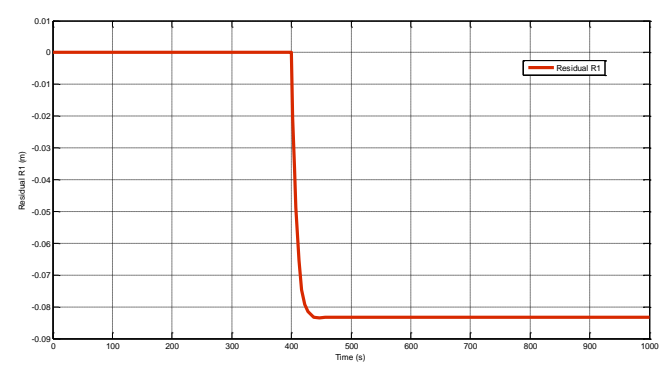

Fig. 21. Residual response in an abnormal operation R1 


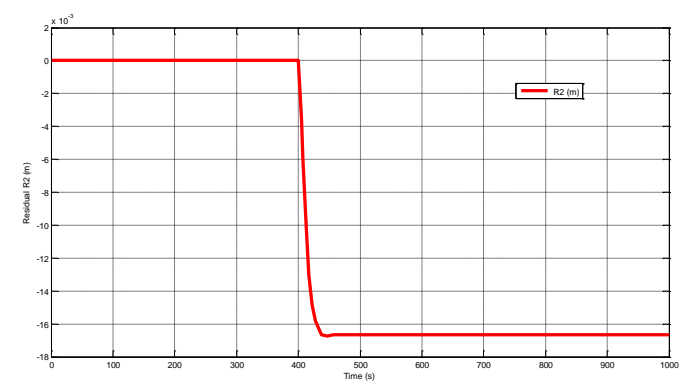

Fig. 22. Residual response in an abnormal operation R2

\section{DISCUSSION AND REMARKS}

Despite the abundance of works dealing with several fault diagnosis methods, the issue of fault monitoring and identification remains open to research. In fact, analytical redundancy and observers approaches, known as classical methods, often have a limited relationship with the structure of the monitored system [23]. In this context, the fundamental concept of parity space is often referred to in order to restrain failure caused by a fault over running using additional physical sensors [24]. As for the parametric estimation approach, the theory involved a continuous estimation of the process parameters through the input/output measurements [25]. All information originate in the black box identification procedure of the recorded information; thereby, it is rather difficult to comprehend which physical system component has been damaged. On the other hand, classical observers require more analytical techniques (such as poles placement, LMIs...) for different gains calculation [26]. Hence, due to the cost of parity space method and the difficulty of implementation of the parametric estimation approach, the graphical observers-based method is widely used techniques for residual generations. Few research works are founded on the building of the graphical observer because of the difficulty of synthesizing a graphical observer [27] [28].

The innovative interest of the present paper consists in the use of directed hypergraph observer for fault monitoring, which may affect the considered system. The Directed Hypergraph tool is considered as a favorite solution capable of building models taking into account the process knowledge. It relies on the qualitative model developed from deep physical understanding of the complex system, thus avoiding the complexity of numerical calculations. A novel diagnosis framework for fault motoring was investigated herein based on energy concept and qualitative reasoning. It aimed to provide a systematic approach to the modeling, analysis and FD schemes. In addition, the construction of Directed Hypergraph Observer used for dynamic systems and any physical domain, provides an estimate of the system state from its model. It aimed particularly to improve the monitoring time and the performances.

Unlike the existing observers [29] [30], the proposed Directed Hypergraph Observer is a graphical model founded on energy transfer between dynamic elements of the studied system. Indeed, the Directed Hypergraph model is taken up to ameliorate the description of interconnected physical parts and to oby the natural causality (Known as integral causality). The future comportment is defined per its current state representation, and subsequent input to a physical process. Energy exchanges among system components can modify its states. At that instant, the Directed Hypergraph representation needs the utilization of natural causality, which enhances the synthesis and the refinement of the proposed graph. The work presented in this paper introduced a control tool for people who develop Directed Hypergraph models, since it is possible to build a graphical observer directly from a Directed Hypergraph model where the matrix calculations can be directly derived from the Directed Hypergraph Observer, avoiding the algebraic manipulation of matrices. From the procedure of the present work it is possible to build an observer with a simpler structure than the structure of the bond graph observer which requires a derived causality assignment for all storage elements in the BG model. Therefore, the causality of the sensors have to be reversed as shown in [31], which means that the calculation of the observer gain is also simpler.

\section{CONCLUSION}

In this paper, qualitative-based graphics have been developed for modelling and fault detection issues, broadly founded on causal and physical principles. The aim was to build an observer model for fault detection algorithms dedicated to engineering system to sustain an understanding of the system performance.

The importance of the directed Hypergraph DH tool lies essentially in its structure, which allows a structural modelling that displays the relationships between the variables of the system by hyperedges. The Directed Hypergraph model can be constructed from mathematical equations, based on the process knowledge using the Bond Graph BG model in integral causality developed from physical understanding of the dynamic systems.

To benefit from the advantages of the Directed Hypergraph tool, we managed to extend the DH model from a pure modelling task to a graphical observer-based fault detection tool. It is therefore necessary, in this case, to reconstruct the state from the information available on the input and output. This observer is often known as a reconstructed or state estimator.

The residues are then obtained by differentiating the measured outputs from their estimates. The 
application of the proposed approach has been achieved through the illustration of a Hydraulic system with two tanks. The Luenberger observer was synthesized based on the Directed Hypergraph DH tool.

Finally, the appearance of undesirable effects is perfectly detected. The simulations validate the effectiveness of the proposed method.

\section{ACKNOWLEDGMENTS}

The authors would like to thank the comments provided by the anonymous reviewers and editor, which helped the authors improve this paper significantly.

\section{REFERENCES}

1. Isermann R. Fault-diagnosis systems: an introduction from fault detection to fault tolerance. Springer Science \& Business Media, 2005.

2. Blanke M, Kinnaert M, Lunze J. Diagnosis and fault-tolerant control. Berlin: Springer, 2006.

3. Serra R, Zanarini G. Complex systems and cognitive processes. Springer Science \& Business Media, 2013.

4. Patton RJ, Frank PM, Clark RN. Issues of fault diagnosis for dynamic systems. Springer Science \& Business Media, 2013.

5. Samantaray AK, Bouamama BO. Model-based process supervision: a bond graph approach. Springer Science \& Business Media, 2008.

6. Borutzky W. Bond graph model-based fault diagnosis of hybrid systems. Cham: Springer International Publishing, 2015.

7. BERGE, Claude. Graphs and hypergraphs. 1973.

8. Klein D, Manning CD. Parsing and hypergraphs. In : New developments in parsing technology. Springer, Dordrecht. 2004 :351-372.

9. Ausiello G, Italiano, GF, Laura L, et al. Structure theorems for optimum hyperpaths in directed hypergraphs. In: International Symposium on Combinatorial Optimization. Springer, Berlin, Heidelberg. 2012:1-14.

10. Gallo G, Longo G, Pallottino S, et al. Directed hypergraphs and applications. Discrete applied mathematics. 1993:42(2-3):177-201.

11. Klamt S, Haus Utz-Uwe. Hypergraphs and cellular networks. PLoS Comput Biol, 2009; 5(5): e1000385.

12. Gazdík I. Modelling systems by hypergraphs. Kybernetes. 2006.

13. Adamski M, Tkacz J. Formal reasoning in logic design of reconfigurable controllers. IFAC Proceedings Volumes. 2012; 45(7):1-6.

14. Khalil W, Merzouki R, Ould-Bouamama B. Hypergraph models for system of systems supervision design. IEEE Transactions on Systems, Man, and Cybernetics-Part A: Systems and Humans. 2012; 42(4):1005-1012. https://doi.org/10.1109/TSMCA.2012.2183350.

15. Haffaf H. Hypergraph for system of systems modeling. Networking and Advanced Systems. 2015: 38 .
16. Abdesselam I, Haffaf H. Hypergraph reconfigurability analysis. IERI Procedia. 2014; 6:22-32.

17. Kotulski L, Sędziwy A, Strug B. Heterogeneous graph grammars synchronization in CAD systems supported by hypergraph representations of buildings. Expert systems with applications. 2014; 41(4):990-998. https://doi.org/10.1016/j.eswa.2013.07.043.

18. Habel A, Kreowski HJ. Some structural aspects of hypergraph languages generated by hyperedge replacement. Annual Symposium on Theoretical Aspects of Computer Science. Springer, Berlin, Heidelberg, 1987:207-219.

19. Minas M. Concepts and realization of a diagram editor generator based on hypergraph transformation. Science of Computer Programming. 2002;44(2): 157-180.

20. Gomand J. Analyse de systèmes multi-actionneurs parallèles par une approche graphique causaleapplication a un processus électromécanique de positionnement rapide. 2008. Thèse de doctorat. Arts et Métiers ParisTech.

21. Borutzky W. Bond graph model-based fault diagnosis of hybrid systems. Cham: Springer International Publishing, 2015.

22. Saoudi G, Harabi R, Abdelkrim MN. Robust fault detection based on bond graph UIO observer. International Journal of Digital Signals and Smart Systems. 2017;1(4):302-322.

23. Ding SX. Advanced methods for fault diagnosis and fault-tolerant control. Springer Berlin Heidelberg. 2021.

24. Wu Y, Zhao D, Liu S, Li, Y. Fault detection for linear discrete time-varying systems with multiplicative noise based on parity space method. ISA transactions. 2021. https://doi.org/10.1016/j.isatra.2021.04.018.

25. Pan J, He W, Shi Y, Hou R, Zhu H. Uncertainty analysis based on non-parametric statistical modelling method for photovoltaic array output and its application in fault diagnosis. Solar Energy. 2021;225, 831-841.

26. Vijay P, Tadé MO, Shao Z. Adaptive observer based approach for the fault diagnosis in solid oxide fuel cells. Journal of Process Control. 2019; 84, 101-114.

27. Atitallah M, El Harabi R, Abdelkrim MN. Unknown input Hamiltonian observers-based fault detection and estimation. Systems, Automation, and Control. 2019: 177-196.

28. Aboub A, El Harabi R, Abdelkrim MN. Causal ordering graph based observers for generating redundant relations. 17th International MultiConference on Systems, Signals \& Devices (SSD). IEEE, 2020:1080-1085).

29. Garai D, El Harabi R, Bacha F. A comparative study of Graphical tools for the Fault Monitoring using HBG and DBH Formalisms. 18th International Multi-Conference on Systems, Signals \& Devices (SSD). IEEE. 2021:124-130).

30. Garai D, El Harabi R, Bacha F. Hamiltonian Bond Graph formalism for generating energetic redundant relations. 17th International Multi-Conference on Systems, Signals \& Devices (SSD). IEEE. 2020:1063-1068)

31. Merahi F, Bouamama BO, Mekhilef S. Bond graph modeling, design and experimental validation of a photovoltaic/fuel cell/electrolyzer/battery hybrid 
power system. International Journal of Hydrogen Energy. 2021;46(47):24011-24027.

https://doi.org/10.1016/j.ijhydene.2021.05.016.

Received 2021-04-18

Accepted 2021-11-22

Available online 2021-11-24

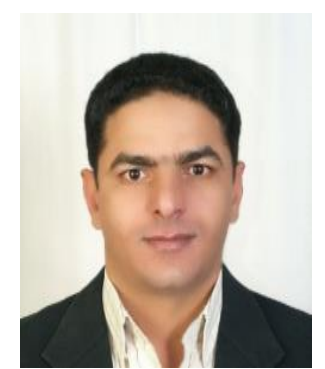

Dhaou GARAI was born in Ben Guerdanne, Tunisia, in 1972. he graduated from the Higher Institute Education And Formation Continue of Tunis, Tunisia, in 1996. he is obtained his master degree in electrical engineering, automatic and robotic systems from higher institute of industrial systems of Gabes (ISSIG), in 2018. Actually, he is preparing her $\mathrm{PhD}$ degree, his research interests are in fault diagnosis, fault tolerant control and complex process supervision with special attention paid to graphical model-based diagnosis (Bond Graph and Directed Hypergraph tools). $\mathrm{He}$ is a member in LISI Laboratory, INSAT, Carthage University, LR11ES26 Carthage, Tunisia.

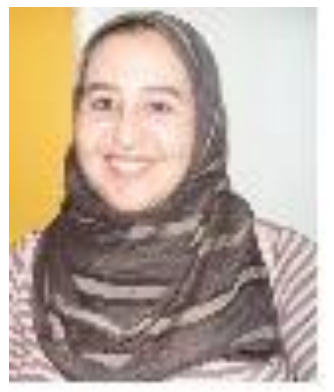

Rafika El HARABI was born in Medenine, Tunisia, in 1982. She graduated from the National Engineering school of Gabes, Tunisia, in 2006, and received the Ph.D. degree in 2011 from both the National Engineering school Of Gabes, Tunisia and University of Lille 1, France, both in Automation and control theory. She is former member in CRIStAL (Research center in Computer Science, Signal and Automatic Control of Lille, UMR 9189). She is a permanent member MACS Laboratory (Modeling, Analyze and Systems Control Laboratory, LR16ES22) at National Engineering School of Gabes, University of Gabes, Tunisia. In 2011, she joined the National Engineering School of Gabes (ENIG), Tunisia, as Assistant in higher education. In 2012, she is Assistant professor in the higher institute of industrial systems of Gabes (ISSIG). She holds, in 2015, a master of research in analysis, design and research in the field of engineering in Education Technology (ACREDITE) from University of Cergy-pontoise (France) in collaboration with University of Geneve, Switzerland and University of Mons, Belgium. She is a permanent member of the steering committee of the digital project at ISSIG. Her research interests are in fault diagnosis, fault tolerant control and complex process supervision with special attention paid to graphical model based diagnosis of chemical processes (Graphical tools). Her new research interests are focused on digital pedagogy and e-Learning.
Faouzi BACHA was born in Ben Guerdanne, Tunisia, in 1964. He received the "Habilitation Universitaire" degree in electrical engineering from the National Institute of engineering, Tunis, in 2008. He is currently a Full Professor with the High National School of Engineers of Tunis, University of Tunis, Tunis. He has authored and coauthored more than 100 publications on power system stability and control, direct power control of synchronous, and induction machines. His research interests include modeling and simulation of electrical machines, power system, and wind energy conversion system, Prof. Bacha is a Member of the Working Group on Wind Energy Application in Tunisia. 